\title{
MODELAGEM MATEMÁTICA APLICADA A EVAPOTRANSPIRAÇÃO DE REFERÊNCIA PARA A CIDADE DE JANUÁRIA (MG)
}

\author{
Romário Santos Madureira \\ Instituto Federal do Norte de Minas Gerais - IFNMG campus Januária e Universidade \\ Estadual de Montes Claros - PPGE Unimontes \\ romario.madureira@ifnmg.edu.br \\ Josué Antunes de Macêdo \\ Instituto Federal do Norte de Minas Gerais - IFNMG campus Januária e Universidade \\ Estadual de Montes Claros - PPGE Unimontes \\ Adriana Martins da Silva Castro \\ Instituto Federal do Norte de Minas Gerais - IFNMG campus Januária \\ adriana.castro@ifnmg.edu.br
}

\section{Resumo}

A Modelagem Matemática proporciona modelar situações cotidianas para uma melhor interpretação dos eventos. Contribui, também, com o desenvolvimento acadêmico e busca por soluções a fim de facilitar problemas de áreas distintas. Para melhor aproveitamento dos recursos hidrícos na região do Norte de Minas Gerais, o presente trabalho objetivou-se em determinar a média mensal da evapotranspiração de referência $\left(E T_{0}\right)$ pelo método Penman Monteith. O saldo de radiação $\left(R_{n}\right)$ correspondeu a 59,0\% do valor médio da radiação solar global. A variação da evapotranspiração oscilou entre os valores $6,82 \mathrm{~mm} /$ dia e $2,57 \mathrm{~mm} /$ dia, obtendo o maior coeficiente de determinação com a radiação líquida em que $R^{2}$ é igual 0,682. No intervalo entre 3,5 e 5,5 mm/dia concentra-se uma amostra considerável das evapotranspirações de referência ocorrentes em Januária (MG).

Palavras-chave: Modelagem Matemática. Evapotranspiração. Radiação.

\section{Abstract}

Mathematical Modeling provides modeling of everyday situations for better interpretation of events. Also, it contributes to academic development and search for solutions in order to facilitate problems in different areas. To make better use of water resources in 
Santos Madureira, R.; Antunes de Macêdo, J; Martins da Silva Castro, A.

the region, this study aimed to determine the monthly mean reference evapotranspiration $\left(E T_{0}\right)$ by the Penman Monteith method. The radiation balance $\left(R_{n}\right)$ corresponded to $59.0 \%$ of the average value of global solar radiation. Evapotranspiration ranged from $6.82 \mathrm{~mm} /$ day to $2.57 \mathrm{~mm} /$ day, obtaining the highest coefficient of determination with net radiation where $R^{2}$ equals 0.682. In the interval between 3.5 and $5.5 \mathrm{~mm} /$ day a considerable sample of the reference evapotranspirations occurring in Januária (MG) is concentrated.

Keywords: Mathematical Modeling. Evapotranspiration. Radiation.

\section{Introdução}

As origens das ideias centrais da Matemática são resultados de processos que procuram entender e explicar os fatos e fenômenos da realidade. O desenvolvimento dessas ideias e sua organização intelectual dão-se a partir de elaborações sobre representações do mundo real.

Na Modelagem Matemática, busca-se a resolução de situações-problemas vivenciadas pela sociedade. Como afirma [6] "Essa metodologia de trabalho estimula a criatividade, aguça o senso crítico, possibilita a discussão de temas de relevância comunitária. O educando passa a ter um papel atuante e socializa os saberes e as dificuldades"(p. 2).

Percebe-se, atualmente, vários problemas no processo de ensino-aprendizagem da Matemática, sendo que das formas de incentivar aprendizagem é a Modelagem. De acordo com [5] "[...] o ensino de Matemática, por meio da Modelagem Matemática, proporciona ao aluno o contato com problemas reais e desenvolve a capacidade de resolvê-los" (p. 82).

A Modelagem Matemática, de acordo com [3] é:

[...] um processo dinâmico utilizado para a obtenção e validação de modelos matemáticos. É uma forma de abstração e generalização com a finalidade de previsão de tendências. A modelagem consiste, essencialmente, na arte de transformar situações da realidade em problemas matemáticos cujas soluções devem ser interpretadas na linguagem usual. (p. 24).

Problemas reais apresentam várias variáveis, sendo que os modelos matemáticos oferecem condições de estudar e fazer previsões, pois permite realizar aproximações da realidade. 
Assim, a Modelagem Matemática surge como estímulo para o aluno interagir de forma prazerosa com a Matemática, usando sua aplicabilidade em soluções e situações do cotidiano, isto fará com que ele perceba na forma do conhecimento técnico-científico a importância do conteúdo estudado em Matemática. Em resumo, a Modelagem Matemática consiste na arte de descrever matematicamente um fenômeno, visando a simulação de sistemas reais a fim de prever o seu comportamento, sendo empregada em diversos campos de estudo, como na área de irrigação, possibilitando a estimativa da evapotranspiração de referência, que é um dos principais elementos climatológicos, fundamentais para a determinação da necessidade hídrica das culturas.

Segundo [8], a evapotranspiração de referência $\left(E T_{0}\right)$ está associada ao processo de perda de água para a atmosfera considerando uma superfície extensa, coberta por grama com altura entre 0,08 e 0,15 $\mathrm{m}$, em crescimento ativo, cobrindo totalmente o solo e sem deficiência de água.

Todavia, a estimativa da $E T_{0}$ através de métodos diretos demanda equipamentos especiais e custos elevados, além de demandar tempo. A utilização de métodos indiretos proporciona resultados satisfatórios, além de minimizar custo e tempo, quando comparado aos métodos diretos. Dentre os métodos indiretos o de Penman-Monteith-FAO é considerado como método padrão para a determinação da $E T_{0}$, como afirma [1].

Este método, além de procurar representar, de maneira consistente, o fenômeno biofísico da evapotranspiração, é alimentado por quase todos os elementos meteorológicos observados em estações meteorológicas, o que torna o método mais próximo da situação real. Porém, devido à grande quantidade de dados utilizados, torna-se na prática o método menos utilizado, devido à sua complexidade. Assim, este estudo teve como propósito discutir a modelagem matemática aplicada à evapotranspiração de referência para a cidade de Januária (MG), utilizando o método padrão da Food and Agriculture Organization of the United Nations (FAO - Organização das Nações Unidas para Alimentação e Agricultura), objetivando esclarecer de maneira prática e eficiente o seu uso.

A Ref. [2], ao discutir atividades de Modelagem na Educação Matemática desenvolvidas na escola, resume que Modelagem Matemática "[...] é um ambiente de aprendizagem no qual os alunos são convidados a problematizar e investigar, por meio da Matemática, situações com referência na realidade" (p. 4).

$\mathrm{Na}$ atualidade, faz-se necessário entender cada vez mais esses fenômenos da realidade. O cálculo infinitesimal é uma ferramenta de grande valia no processo de Modelagem Matemática para o desenvolvimento produtivo, satisfatório e eficiente da formalização e transição do mundo matemático para compilação de uma linguagem acessível à coletividade.

Um fato que tem preocupado a população da região de Januária (MG), também 
conhecida como região do Médio São Francisco, é o déficit de recursos hídricos que assola tal região de forma mais rígida a cada ano subsequente.

O clima da região é tropical com transição para semiárido, graças à deficiência de água no solo e ao forte calor, a vegetação de Januária é xeromorfa, ou seja, tem formas adaptadas à seca, composta por cerrado, matas secas, caatinga e veredas cobertas de buritis. A economia concentra-se na agricultura e na pecuária.

A água é um dos principais elementos no processo de nutrição das plantas e o desenvolvimento de técnicas para sua conservação no solo requer o conhecimento dos processos envolvidos na evaporação e na dinâmica da água no solo.

A evaporação tem sido pesquisada sob diversos aspectos [9], principalmente relacionada com a evapotranspiração e a influência das condições meteorológicas.

A evaporação tem influência de vários fatores, dentre eles os mais importantes são a textura, o teor de umidade e a temperatura do solo, a intensidade da radiação, a temperatura do ar e a intensidade dos ventos superficiais. A determinação das condições de superfície são complexas, imprecisas e trabalhosas (controle de umidade do ar, velocidade do vento e radiação).

Sendo a água um recurso importante para a sobrevivência e manutenção da região, torna-se viável utilizar a Matemática para interpretar como a radiação solar interfere na evapotranspiração na região do Médio São Francisco.

Poucos períodos de chuvas e altas temperaturas favorecem para a região possuir um Índice Ultravioleta (IUV) de 8 a 10, considerado muito alto, de acordo com o Centro de Previsão do Tempo e Estudos Climáticos do INPE [7].

A radiação solar é um dos fatores que contribuem para a ocorrência do processo de evapotranspiração. Busca-se relevância no estudo analítico matemático da radiação solar nos últimos cincos anos para mostrar uma previsão para anos subsequentes, na intenção de colaborar no planejamento de ações para melhoria dos recursos hídricos da região.

Nesse sentido, a Matemática, através da Modelagem, começa a considerar o contexto social e cultural que o aluno está inserido. Neste trabalho, a Modelagem contribuiu para o entendimento da influência da radiação solar na estimativa da evapotranspiração.

De acordo com [4], a água pode ser transferida do solo para a atmosfera através da transpiração e da evaporação. Esses dois processos são conhecidos como evapotranspiração. O processo de evaporação da água representa 50\% da evapotranspiração. Assim justifica-se pesquisar os fatores responsáveis pela evaporação, tendo em vista a fornecer meios da conservação de maior quantidade de água no solo.

Portanto, de forma estimulante, interessante para o aumento do conhecimento e ao mesmo tempo com a intenção de contribuir com a coletividade, este trabalho servirá de consulta para auxiliar profissionais da área e os órgãos públicos sobre futuras decisões 
Santos Madureira, R.; Antunes de Macêdo, J; Martins da Silva Castro, A.

para quantificar os recursos hídricos da região do Médio São Francisco.

\section{Metodologia e Execução}

A pesquisa foi desenvolvida na cidade de Januária (MG), região do Médio São Francisco, sob coordenadas geográficas (Lat.: $15^{\circ} 45^{\prime} \mathrm{S}$; Long.: $44^{\circ} \mathrm{W}$ e altitude $473,71 \mathrm{~m}$ ). O Clima é tropical com transição para semiárido, sendo que a temperatura máxima atinge $38^{\circ} \mathrm{C}$, a mínima $12,6^{\circ} \mathrm{C}$ e a média anual de $26,3^{\circ} \mathrm{C}$. Os dados foram coletados do Banco de Dados Meteorológicos para Ensino e Pesquisa (BDMEP), disponibilizados pelo Instituto Nacional de Meteorologia (INMET) e aferidos pela estação meteorológica situada no Instituto Federal de Educação, Ciência e Tecnologia do Norte Minas Gerais, Campus Januária, no período de janeiro de 2009 até dezembro de 2013.

Inicialmente, a estimativa da evapotranspiração $\left(E T_{0}\right)$ seria diária, no entanto, devido a indisponibilidade de alguns dados diários, foi necessário optar por determinar a média mensal para o período estudado.

O cálculo da evapotranspiração de referência $\left(E T_{0}\right)$ pelo modelo Penman-Monteith (Padrão FAO) considera a resistência estomática de $70 \mathrm{~s} / \mathrm{m}$, albedo de 0,23 e a altura da grama fixada em 0,12 m, que de acordo com a Ref. [1] pode ser obtida pela equação (2.1) abaixo:

$$
E T_{0}=\frac{0,408 \Delta\left(R_{n}-G\right)+\gamma\left(\frac{900 U_{2}}{T+273}\right)\left(e_{s}-e_{a}\right)}{\Delta+\gamma\left(1+0,34 U_{2}\right)} .
$$

Em que:

$E T_{0}$ : evapotranspiração de referência, $\mathrm{mm} /$ dia;

$R_{n}$ : radiação líquida ou saldo de radiação, $\left(M J / m^{2}\right.$ dia $)$;

$G$ : fluxo de calor no solo, $\left(M J / m^{2}\right.$ dia);

$T$ : temperatura média do ar diária medida a $2 m$ de altura, $\left({ }^{\circ} \mathrm{C}\right)$;

$U_{2}$ : velocidade do vento medido a $2 \mathrm{~m}$ de altura, $(\mathrm{m} / \mathrm{s})$;

$\triangle$ : declividade da curva de pressão de vapor, $\left(\mathrm{kPa} /{ }^{\circ} \mathrm{C}\right)$;

$e_{s}$ : pressão de vapor saturado do ar, $(k P a)$;

$e_{a}$ : pressão de vapor do ar na altura $z,(k P a)$;

$\gamma$ : é o fator psicométrica, $(M J K / g)$.

Na impossibilidade de acesso a algumas variáveis, inexistência doutras, foram utilizadas as parametrizações proposta pela FAO 56 e descritas na Ref. [1], sendo o cálculo da radiação líquida $\left(R_{n}\right)$, variável de maior complexidade dentre as presentes na equação. Para determinar $R_{n}$ foi necessário determinar a radiação no topo da atmosfera $\left(R_{a}\right)$, 
radiação incidente em um dia limpo $\left(R_{s o}\right)$, balanço de ondas longas $\left(R_{n l}\right)$, balanço de ondas curtas $\left(R_{n s}\right)$, sendo utilizadas as seguintes equações:

$$
R_{a}=\frac{24(60)}{\pi} G_{s c} d\left[\varpi_{s} \sin (\varphi) \sin (\delta)+\cos (\varphi) \cos (\delta) \sin \left(\varpi_{s}\right)\right]
$$

Em que:

$R_{a}=$ Radiação extraterrestre $\left(M J / m^{2}\right.$ dia);

$G_{s c}=$ constante solar $=0,0820\left(M J / m^{2}\right.$ dia $)$;

$d=$ Distância relativa inversa Terra-Sol;

$\varpi_{s}=$ Ângulo horário pôr do Sol (radiano);

$\varphi=$ Latitude local (radiano);

$\delta=$ Declinação solar (radiano).

A distância relativa Terra-Sol, é determinada por:

$$
D r=1+0,033 \cos \left(\frac{2 \pi}{365} D J\right) .
$$

Em que:

$D J=$ Dia Juliano.

A declinação solar $(\delta)$, é dada por:

$$
\delta=0,409 \sin \left(\frac{2 \pi}{365} D J-1,39\right) .
$$

O ângulo horário pôr do Sol $\left(\omega_{s}\right)$ é dado por:

$$
\varpi_{s}=\arccos [-\tan (\varphi) \tan (\delta)] .
$$

A radiação solar em céu totalmente limpo $\left(R_{s o}\right)$ foi calculada por:

$$
R_{\text {so }}=\left(0,75+2 \times 10^{-5} Z\right) R_{a} .
$$

Em que:

$R_{s o}=$ Radiação solar em céu totalmente limpo, $M J / m$ dia;

$Z=$ Altitude local, metros.

Para o cálculo do Balanço de ondas longas $\left(R_{n l}\right)$ foi usada a seguinte equação:

$$
R_{n l}=\sigma\left[\frac{\left(T_{\max }+273\right)^{4}+\left(T_{\min }+273\right)^{4}}{2}\right]\left(0,34-0,14 \sqrt{e_{a}}\right)\left(1,35 \frac{R_{s}}{R_{s o}}-0,35\right) .
$$


Em que:

$R_{n l}=$ Balanço de ondas longas $\left(M J / m^{2}\right.$ dia);

$\sigma=$ constante de Stefan-Boltzamann $\left(4,9 \cdot 10^{-9} M J K^{-4} m^{-2} \mathrm{dia}^{-1}\right)$;

$T_{\max }=$ máxima temperatura $\left({ }^{\circ} \mathrm{C}\right)$;

$T_{\text {min }}=$ mínima temperatura $\left({ }^{\circ} \mathrm{C}\right)$;

$e_{a}=$ Pressão de vapor atual $(k P a)$;

$R_{s}\left(R_{s o}\right)^{-1}=$ Relação da radiação solar incidente.

Em seguida, foi estimado o balanço de ondas curtas $\left(R_{n s}\right)$, pela seguinte equação:

$$
R_{n s}=(1-\alpha) R_{s}
$$

Em que:

$\alpha=$ Albedo da superfície, decimal. Foi utilizado 0,23, conforme recomenda-se a FAO.

E, finalmente, a partir dos dados anteriores,

$$
R_{n}=R_{n s}-R_{n l}
$$

Em que:

$R_{n}=$ Radiação líquida, $M J / m$ dia.

Os procedimentos detalhados para obtenção dos parâmetros da expressão, foram executadas de acordo com a FAO 56 [1], para uma maior confiabilidade da pesquisa. O valor estimado da $E T_{O}$ configura média diária mensal, semelhante aos dados de temperatura do ar, velocidade do vento, horas de insolação, declividade da curva de pressão de saturação do vapor d'água, pressão de saturação do vapor d'água, pressão atual de vapor d'água e umidade relativa do ar.

Para determinar a média aritmética da radiação extraterrestre $\left(R_{a}\right)$, radiação solar no céu totalmente limpo $\left(R_{s o}\right)$, ângulo pôr do Sol $\left(\omega_{s}\right)$, declinação solar e distância relativa do Sol, optou-se pelos dias Julianos $(16,47,75,105,136,167,197,228,259,289,320$ e 350) existindo um ajuste em 2012 (16, 46, 76, 106, 137, 168, 198, 229, 260, 270, 321 e 351) por ser ano bissexto. Tais números apresentam justamente o valor da média mensal escolhida quando cada variável citada acima foi calculada diariamente, ou seja, a média do somatório dos valores diários aparecia nas linhas dos dias referidos.

Através da radiação líquida obtida, foi estimado a $E T_{0}$ e as análises das variáveis foram feitas utilizando um software de planilha eletrônica através de regressão linear.

Em conjunto, foi estimado o balanço de radiação e a frequência de ocorrência da evapotranspiração de referência na cidade de Januária (MG). Para isso, adotou-se o método de Kimbal, pela equação: 
Santos Madureira, R.; Antunes de Macêdo, J; Martins da Silva Castro, A.

$$
f=\frac{m}{n+1}
$$

Em que:

$f=$ frequência de ocorrência da evapotranspiração de referência;

$m=$ ordem do evento, ou seja, número de vezes que uma evapotranspiração de

referência do mesmo valor ocorreu ou foi ultrapassada em um determinado período de tempo;

$n=$ número de dados analisados.

Nessa equação leva-se em conta que a estimativa da frequência de ocorrência de um evento climatológico nunca será totalmente verdadeira.

Assim, foi possível obter a regressão linear entre evapotranspiração referencial e frequência de ocorrência afim de prever a probabilidade da evapotranspiração de referência média mensal para os anos subsequentes.

\section{Resultados Principais}

Na Fig. 3 é apresentada uma regressão polinomial entre os valores diários (01/01/2009) a (31/12/2013) do ângulo horário pôr do Sol e radiação extraterrestre, apresentado um coeficiente de determinação $R^{2}=0,998$ indicando uma dependência significativa entre as variáveis, pois está bem próximo de 1,000.

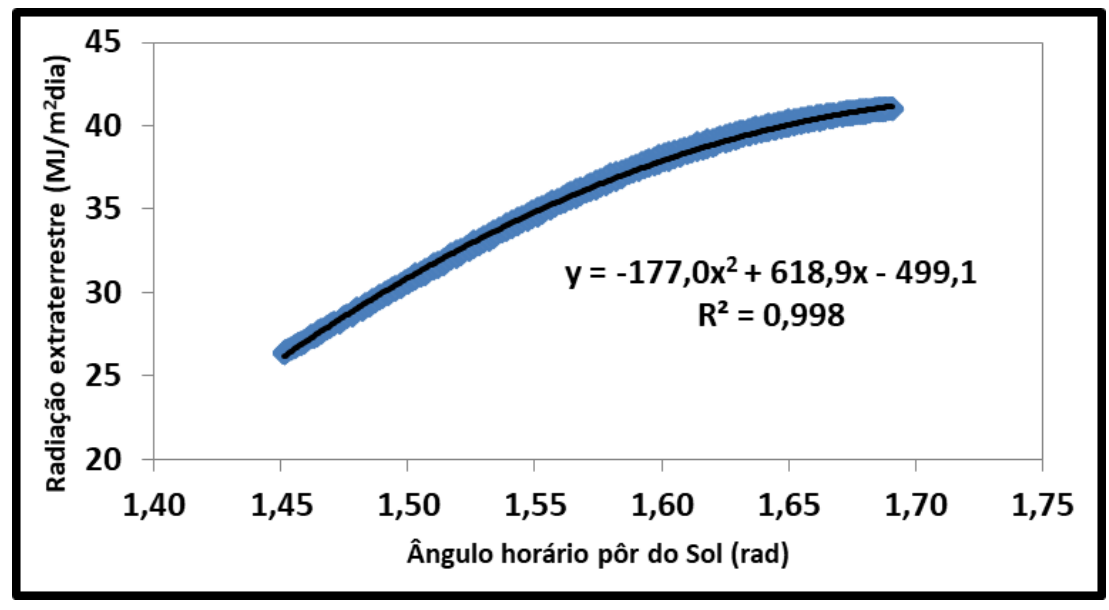

Figura 1: Regressão polinomial de ordem 2 com dados diários entre ângulo horário pôr do Sol $\left(\omega_{s}\right)$ e radiação extraterrestre $\left(R_{a}\right)$ para a região de Januária (MG).

Fonte: Dados da pesquisa 
Com os dados brutos e um nível de confiança $\alpha=0,05$, determinou-se através de um software estatístico os cálculos da análise de variância, cujos dados são resumidos na Tabela 1.

\begin{tabular}{lllll}
\hline & $\begin{array}{l}\text { Graus de Liber- } \\
\text { dade }(g l)\end{array}$ & $\begin{array}{l}\text { Somatório dos } \\
\text { Quadrados }(S Q)\end{array}$ & $\begin{array}{l}\text { Média dos Qua- } \\
\text { drados }(M Q)\end{array}$ & F \\
\hline Regressão & 2 & 10352,0409 & 5176,0204 & 129077,8155 \\
Resíduo & 363 & 14,5576 & 0,0401 & \\
Total & 365 & 10366,5985 & & \\
\hline
\end{tabular}

Tabela 1: Análise de variância (ANOVA) para a regressão polinomial de ordem 2 com dados diários entre ângulo horário pôr do Sol $\left(\omega_{s}\right)$ e radiação extraterrestre $\left(R_{a}\right)$ para a região de Januária ( $\mathrm{MG}$ ).

Avaliando a Fig. 2, observa-se que os resíduos parecem ser independentes e que a variância pode ser considerada aproximadamente constante.

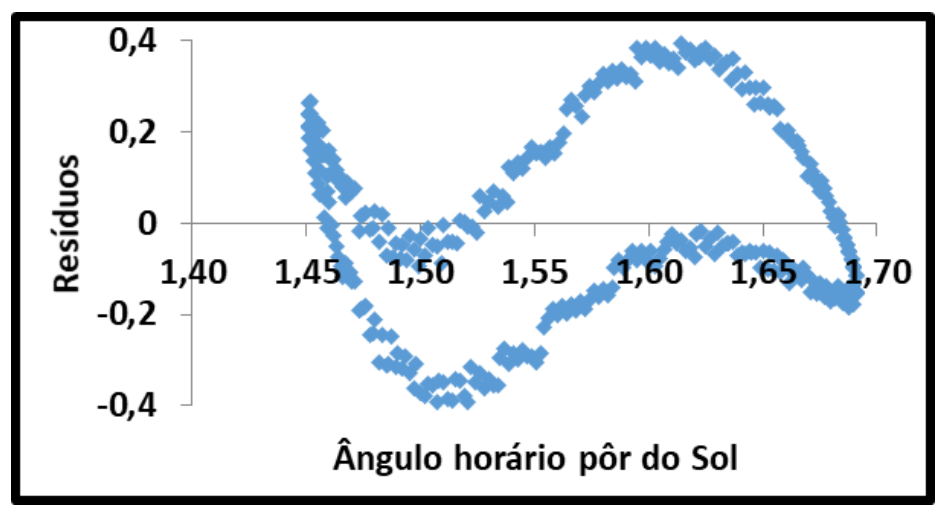

Figura 2: Plotagem dos resíduos para a regressão polinomial de ordem 2 com dados diários entre ângulo horário pôr do Sol $\left(\omega_{s}\right)$ e radiação extraterrestre $\left(R_{a}\right)$ para a região de Januária (MG).

Fonte: Dados da pesquisa

Após o cálculo dos parâmetros e dos resíduos foi possível verificar as hipóteses da regressão, utilizando a estatística de teste $F$ de Fisher, em que

$$
F=\frac{M Q_{\text {Regressão }}}{M Q_{\text {Resíduo }}}
$$

Nesse caso a equação de Regressão polinomial de ordem 2, possui a forma $y=$ $\beta_{1} x^{2}+\beta_{2} x+\alpha$. Definindo-se a hipótese nula $\left(H_{0}\right)$ e a hipótese alternativa $\left(H_{1}\right)$ como 
$H_{0}: \beta_{1}=\beta_{2}=0$ (não há correlação significativa entre x e y),

$H_{1}$ : há pelo menos um $\beta_{i} \neq 0$ (há correlação significativa entre x e y),

e substituindo os dados da Tabela 1 na equação 3.1, obtém-se $F=129077,8155$.

Se $F$ calculado for maior que o valor tabelado para $(p-1)$ graus de liberdade no numerador e $(n-p)$ graus de liberdade no denominador $\left(F_{(p-1, n-p)}\right)$ então a hipótese nula pode ser descartada. Nesse caso tem-se evidência estatística de que há correlação entre as variáveis. Consultando o valor tabelado, considerando $\alpha=0,05$, obtém-se $F_{(2,363)}=3,9201$.

Como $F_{\text {Calculado }}=129077,81553 \gg F_{\text {tabelado }}=3,9201$, rejeita-se a hipótese nula, logo a equação de regressão explica, com alto grau de fidedignidade, os dados encontrados.

A Fig. 3 proporciona a visualização que justifica o uso de valores mensais (2009 2013) para calcular a radiação extraterrestre $\left(R_{a}\right)$ em função do ângulo horário pôr do Sol, uma vez que o $R^{2}$ é igual ao encontrado na equação polinomial da Fig. 3. Sendo assim, na região de Januária (MG) pode-se substituir a equação (2.2) pela equação mostrada na Fig. 3, facilitando o cálculo da radiação extraterrestre.

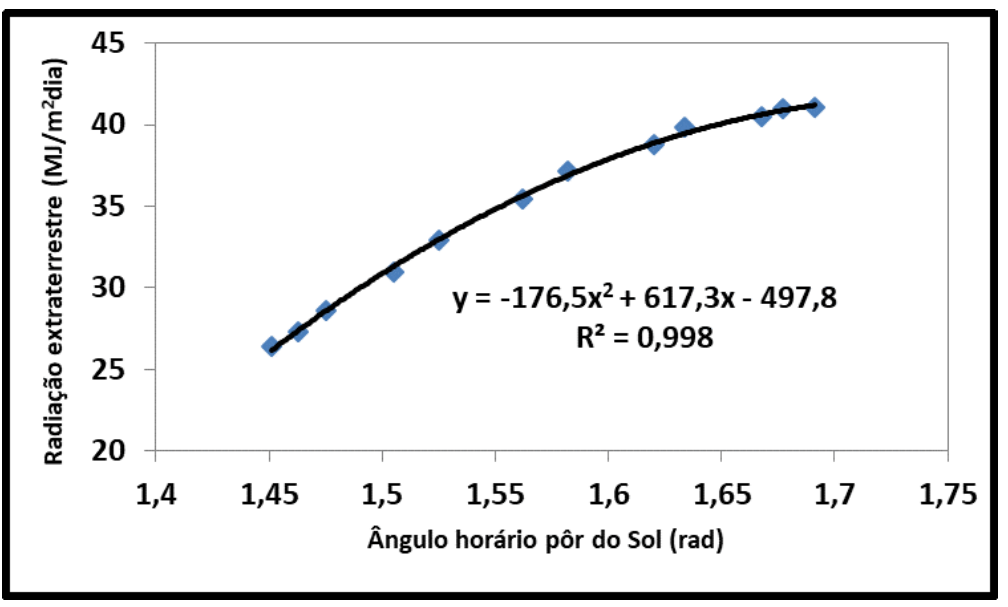

Figura 3: Regressão polinomial de ordem 2 com dados mensais entre Ângulo horário pôr do Sol $\left(\omega_{s}\right)$ e radiação extraterrestre $\left(R_{a}\right)$ para a região de Januária $(\mathrm{MG})$.

Fonte: Dados da pesquisa

A análise de variância para os dados da Fig. 3 são resumidos na Tabela 2, considerandose um nível de confiança $\alpha=0,05$. 
Santos Madureira, R.; Antunes de Macêdo, J; Martins da Silva Castro, A.

\begin{tabular}{lllll}
\hline & $\begin{array}{l}\text { Graus de Liber- } \\
\text { dade }(g l)\end{array}$ & $\begin{array}{l}\text { Somatório dos } \\
\text { Quadrados }(S Q)\end{array}$ & $\begin{array}{l}\text { Média dos Qua- } \\
\text { drados }(M Q)\end{array}$ & \\
\hline Regressão & 2 & 337,9693 & 168,9847 & 3050,2653 \\
Resíduo & 9 & 0,4986 & 0,0554 & \\
Total & 11 & 338,4679 & & \\
\hline
\end{tabular}

Tabela 2: Análise de variância (ANOVA) para a regressão polinomial de ordem 2 com dados mensais entre ângulo horário pôr do Sol $\left(\omega_{s}\right)$ e radiação extraterrestre $\left(R_{a}\right)$ para a região de Januária (MG).

Na Fig. 4 pode-se ver a plotagem dos resíduos, que estão no intervalo $(-0,4,0,4)$.

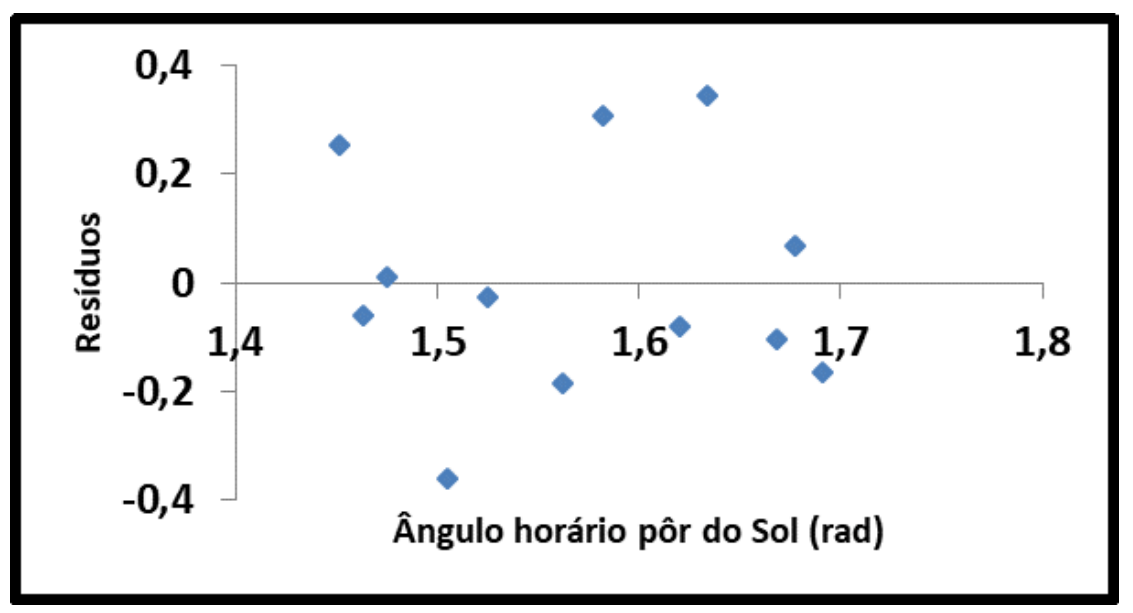

Figura 4: Plotagem dos resíduos para a regressão polinomial de ordem 2 com dados mensais entre ângulo horário pôr do Sol $\left(\omega_{s}\right)$ e radiação extraterrestre $\left(R_{a}\right)$ para a região de Januária (MG).

Fonte: Dados da pesquisa

Como anteriormente, utilizamos a estatística de teste $F$ de Fisher e a equação (3.1). Definindo-se a hipótese nula $\left(H_{0}\right)$ e a hipótese alternativa $\left(H_{1}\right)$ como

$H_{0}: \beta_{1}=\beta_{2}=0$ (não há correlação significativa entre $\mathrm{x}$ e y),

$H_{1}$ : há pelo menos um $\beta_{i} \neq 0$ (há correlação significativa entre x e y),

e consultando o valor tabelado, considerando $\alpha=0,05$, obtém-se $F_{(2,9)}=4,2665$.

Como $F$ Calculado $=3050,2653 \gg F_{\text {tabelado }}=4,2665$, pode-se rejeitar a hipótese nula, admitindo-se a equação de regressão como um bom modelo a ser utilizado. 
O ângulo horário pôr do Sol varia de 1,45 a 1,69 rad, enquanto a radiação extraterrestre 26, 4 a $41 \mathrm{MJ} / \mathrm{m}^{2}$ dia. Para uma melhor análise, a Fig. 5 mostra o balanço da radiação.

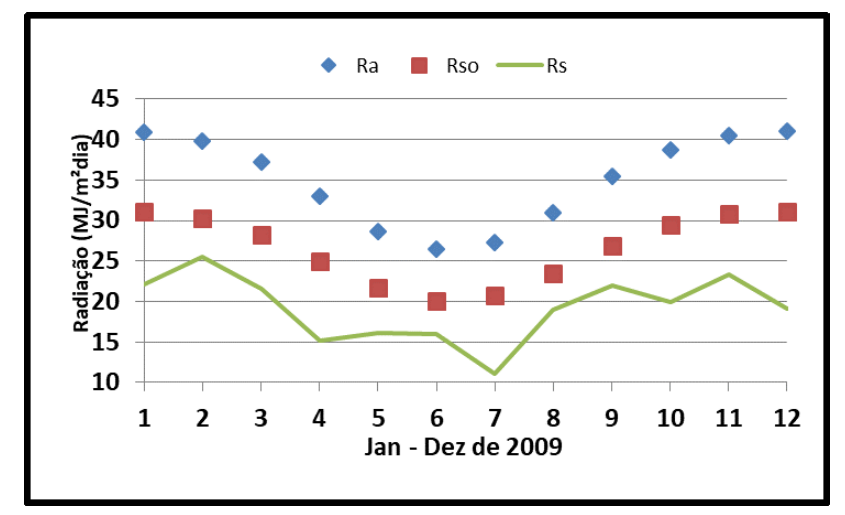

Figura 5: Comportamento anual da Radiação no topo da atmosfera $\left(R_{a}\right)$, para um dia de céu totalmente limpo $\left(R_{s o}\right)$ e Radiação solar global incidente $\left(R_{s}\right)$ para região do Médio São Francisco.

Fonte: Dados da pesquisa

A Fig. 5 apresenta variações esperadas de acordo com as estações do ano, ou seja, a radiação tem índices maiores nos meses de novembro a fevereiro, caracterizando maior parte do verão e índices menores de maio a julho equivalente ao inverno no Hemisfério Sul.

A radiação no topo da atmosfera $\left(R_{a}\right)$ e para um céu totalmente limpo $\left(R_{s o}\right)$ se comporta igual, para todos os anos observados. No entanto, a radiação solar global incidente $\left(R_{s}\right)$ sofre alteração, devido às horas de insolação em cada mês serem distintas. A Fig. 6 evidencia tal afirmação.

Na Fig. 7, estão apresentadas as variações dos componentes do balanço de radiação. Observou-se que as variações do balanço de radiação $\left(R_{n}\right)$ estiveram diretamente associados às variações da radiação solar global (Fig. 3). Analisando estas figuras, verifica-se que a radiação solar global é o principal componente do balanço de radiação, determinando assim o comportamento dos demais termos do balanço.

A radiação solar global variou de $11,06 \mathrm{MJ} / \mathrm{m}^{2}$ dia no mês de julho até um valor máximo de 23, $30 \mathrm{MJ} / \mathrm{m}^{2}$ dia no mês de novembro (Fig. 5). O saldo de radiação $\left(R_{n}\right)$ atingiu o seu valor máximo de $16,11 \mathrm{MJ} / \mathrm{m}^{2}$ dia e valor mínimo de $6,41 \mathrm{MJ} / \mathrm{m}^{2}$ dia. O saldo de radiação representou cerca de $59 \%$ da radiação solar global para região de Januária (MG), no período considerado, conforme pode se ver na Fig. 5.

Dentre as variáveis necessárias para estimar a evapotranpiração de referencia $\left(E T_{0}\right)$, a radiação liquída $\left(R_{n}\right)$ apresentou o maior coeficiente de determinação $\left(R^{2}\right)$ seguida 
Santos Madureira, R.; Antunes de Macêdo, J; Martins da Silva Castro, A.

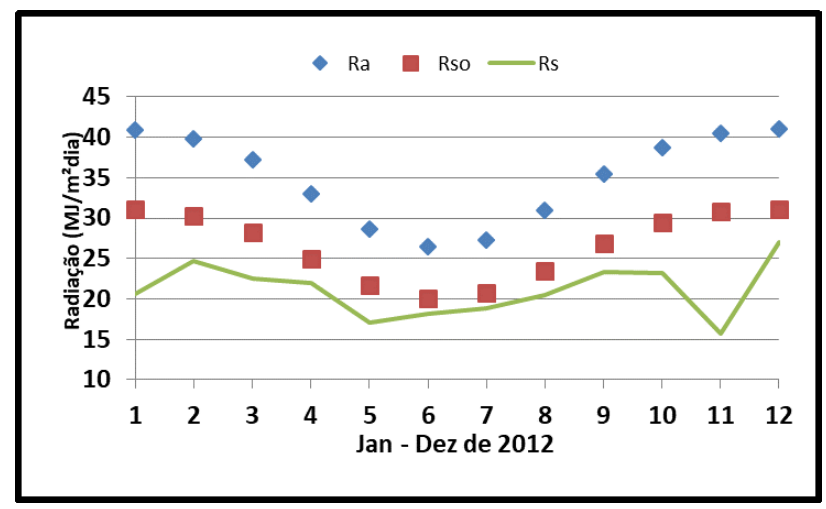

Figura 6: Comportamento anual da Radiação no topo da atmosfera $\left(R_{a}\right)$, para um dia de céu totalmente limpo $\left(R_{s o}\right)$ e Radiação solar global incidente $\left(R_{s}\right)$ na região do Médio São Francisco em ano bissexto.

Fonte: Dados da pesquisa

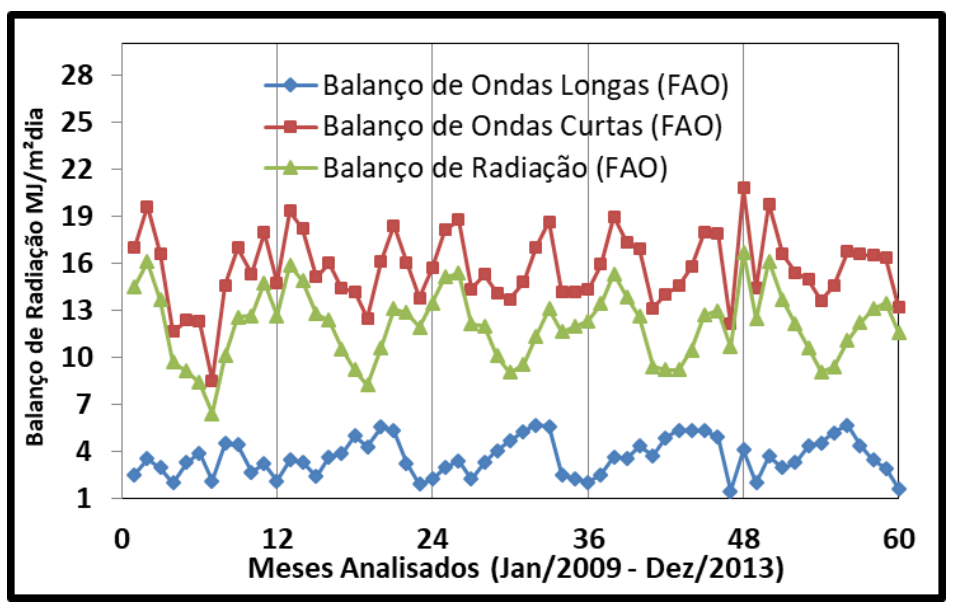

Figura 7: Variação dos componentes da radiação solar para região do Médio São Francisco

Fonte: Dados da pesquisa

da temperatura do ar, ambas quando correlacionadas com a $E T_{0}$, como pode se ver na Fig. 8 e na Fig. 10.

Com os dados da Fig. 8, foi possível determinar a análise de variância, cujos dados são resumidos na Tabela 3, considerando-se um nível de confiança $\alpha=0,05$. 
Santos Madureira, R.; Antunes de Macêdo, J; Martins da Silva Castro, A.

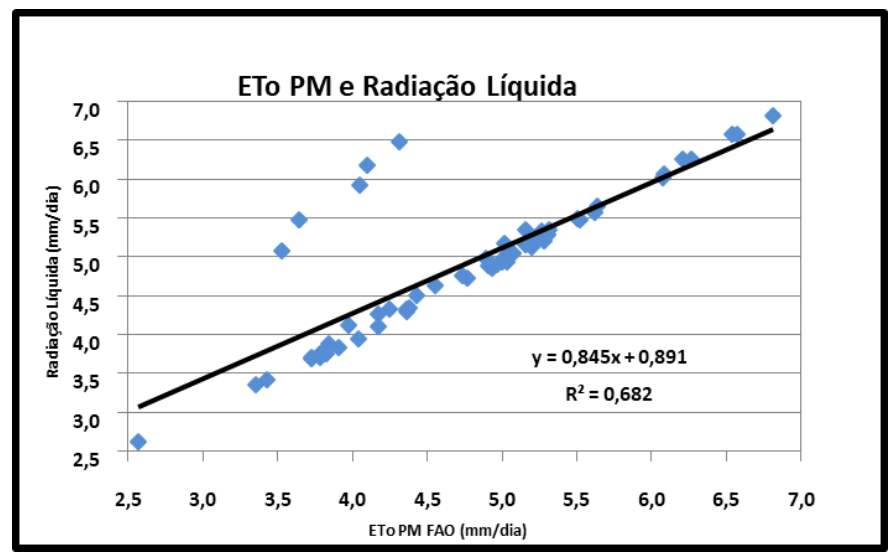

Figura 8: Regressão linear da evapotranspiração com a variável radiação líquida.

Fonte: Dados da pesquisa

\begin{tabular}{lllll}
\hline & $\begin{array}{l}\text { Graus de Liber- } \\
\text { dade }(g l)\end{array}$ & $\begin{array}{l}\text { Somatório dos } \\
\text { Quadrados }(S Q)\end{array}$ & $\begin{array}{l}\text { Média dos Qua- } \\
\text { drados }(M Q)\end{array}$ & \\
\hline Regressão & 2 & 34,2114 & 34,2114 & 124,9047 \\
Resíduo & 58 & 15,8871 & 0,2739 & \\
Total & 59 & 50,0985 & & \\
\hline
\end{tabular}

Tabela 3: Análise de variância (ANOVA) para a regressão linear da evapotranspiração com a variável radiação líquida

A Fig. 9 mostra o gráfico dos resíduos, no qual pode-se ver que a maioria estão próximos de zero.

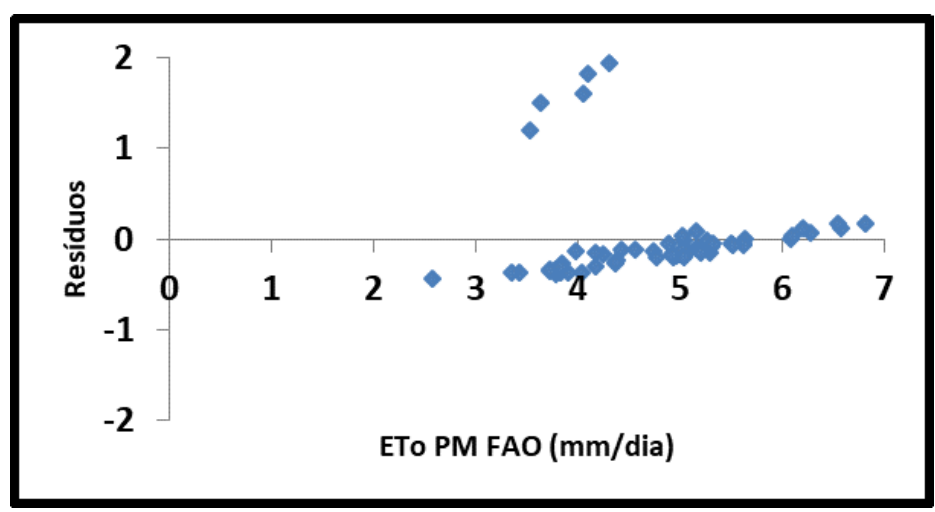

Figura 9: Plotagem dos resíduos para a regressão linear da evapotranspiração com a variável radiação líquida.

Fonte: Dados da pesquisa 
Como nesse caso trata-se de uma equação de regressão linear, que assume a forma $y=\beta x+\alpha$, utilizando a estatística de teste $F$ de Fisher, a equação (3.1) e definindo-se a hipótese nula $\left(H_{0}\right)$ e a hipótese alternativa $\left(H_{1}\right)$ como

$H_{0}: \beta=0$ (não há correlação significativa entre $\mathrm{x}$ e $\mathrm{y}$ ),

$H_{1}: \beta \neq 0$ (há correlação significativa entre $\mathrm{x}$ e y),

e consultando o valor tabelado, considerando $\alpha=0,05$, obtém-se $F_{(1,58)}=4,0012$.

Como $F$ Calculado $=124,9047>F_{\text {tabelado }}=4,0012$, pode-se rejeitar a hipótese nula, admitindo-se que a equação linear representa um bom modelo para a determinação da evapotranspiração.

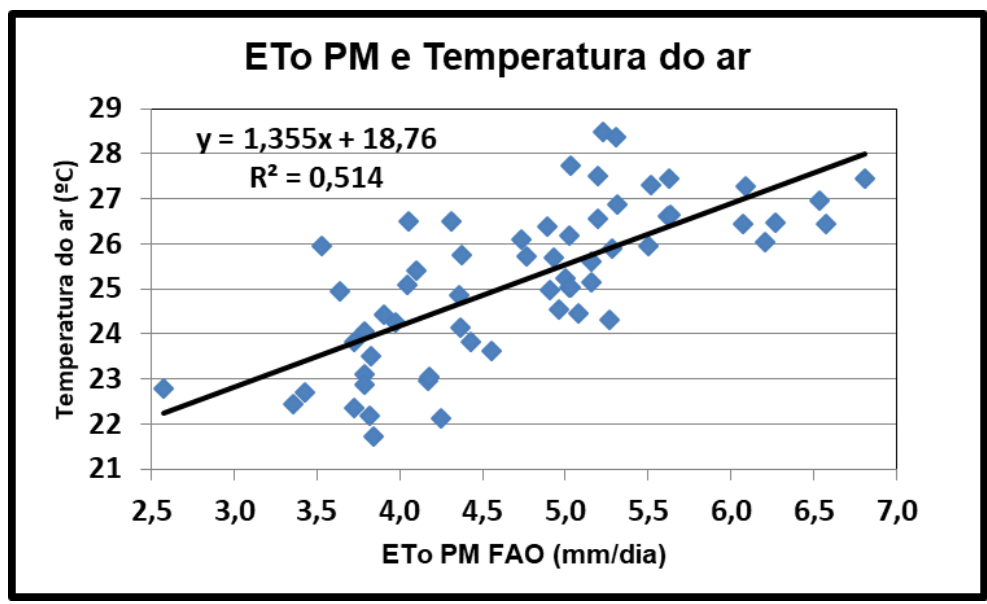

Figura 10: Regressão linear da evapotranspiração com a variável temperatura do ar. Fonte: Dados da pesquisa

Considerando-se um nível de confiança $\alpha=0,05$ e os dados da Fig. 10 foi possível determinar a análise de variância, cujos dados são resumidos na Tabela 4.

\begin{tabular}{llllll}
\hline & $\begin{array}{l}\text { Graus de Liber- } \\
\text { dade }(g l)\end{array}$ & $\begin{array}{l}\text { Somatório dos } \\
\text { Quadrados }(S Q)\end{array}$ & $\begin{array}{l}\text { Média dos Qua- } \\
\text { drados }(M Q)\end{array}$ & F \\
\hline Regressão & 2 & 88,0762 & 88,0762 & 61,9890 \\
Resíduo & 58 & 83,0476 & 1,4319 & \\
Total & 59 & 171,1238 & & \\
\hline
\end{tabular}

Tabela 4: Análise de variância (ANOVA) para a regressão linear da evapotranspiração com a variável radiação líquida. 
Santos Madureira, R.; Antunes de Macêdo, J; Martins da Silva Castro, A.

A Fig. 11 mostra o gráfico dos resíduos que estão compreendidos no intervalo $(-3,3)$.

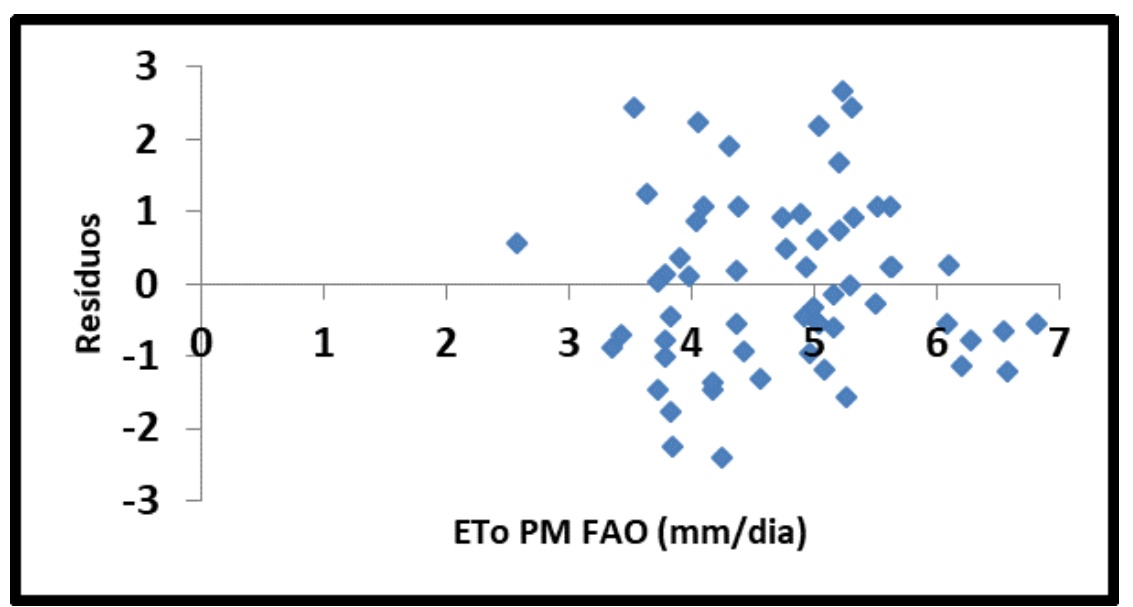

Figura 11: Plotagem dos resíduos para a regressão linear da evapotranspiração com a variável temperatura do ar.

Fonte: Dados da pesquisa

Utilizando a estatística de teste $F$ de Fisher para uma equação linear, a equação (3.1) e definindo-se a hipótese nula $\left(H_{0}\right)$ e a hipótese alternativa $\left(H_{1}\right)$ como

$H_{0}: \beta=0$ (não há correlação linear significativa entre $\mathrm{x}$ e $\mathrm{y}$ ),

$H_{1}: \beta \neq 0$ (há correlação linear significativa entre $\mathrm{x}$ e y),

e consultando o valor tabelado, considerando $\alpha=0,05$, obtém-se $F_{(1,58)}=4,0012$.

Como $F$ Calculado $=124,9047 \gg F_{\text {tabelado }}=4,0012$, pode-se rejeitar a hipótese nula, admitindo-se que a equação linear representa um modelo razoável para a determinação da evapotranspiração.

A Fig. 12 mostra a estimativa dos 60 meses analisados para determinar a evapotranspiração de referência $(\mathrm{mm} / \mathrm{dia})$, cada ponto equivale a um mês e sua média evaporativa diária.

Em janeiro de 2013, foi registrado a maior média de $E T_{0}$, chegando $6,82 \mathrm{~mm} /$ dia e o valor mínimo de $2,57 \mathrm{~mm} /$ dia foi em julho de 2009 . 
Santos Madureira, R.; Antunes de Macêdo, J; Martins da Silva Castro, A.

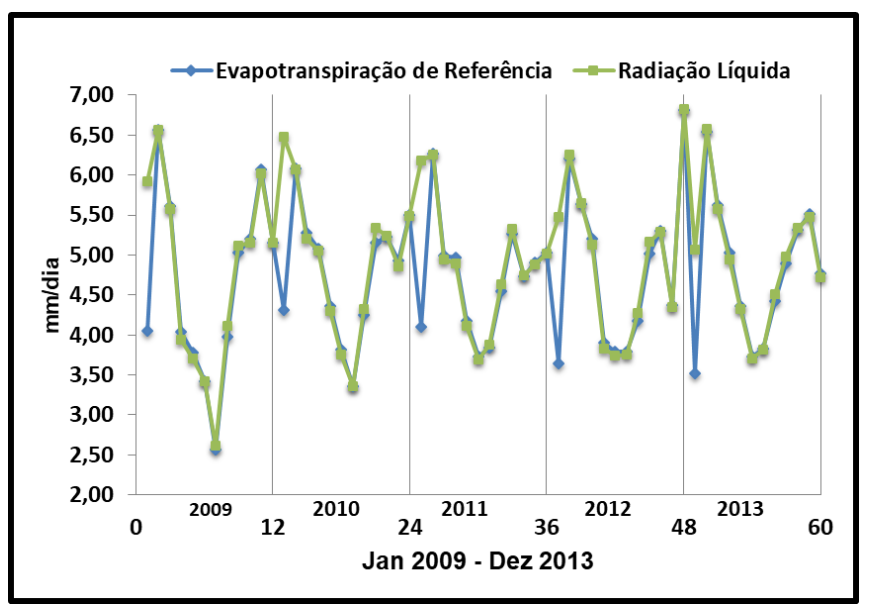

Figura 12: Estimativa mensal da evapotranspiração de referência $(\mathrm{mm} / \mathrm{dia})$ com a variável de maior correlação linear.

Fonte: Dados da pesquisa

\section{Conclusão}

A radiação extraterrestre $\left(R_{a}\right)$ e para céu totalmente limpo $\left(R_{s o}\right)$ são constantes independentes do ano, sendo a equação (2.2) podendo ser substituída pela equação mostrada na Fig. 2. O saldo de radiação $\left(R_{n}\right)$ correspondeu a $59 \%$ do valor médio da radiação solar global. A variável que tem maior contribuição na evapotranspiração de referência na região de Januária $(\mathrm{MG})$ é a radiação líquida $\left(R_{n}\right)$ com um coeficiente de determinação $R^{2}=0,682$.

A frequência de ocorrência de evapotranspiração de referência foi analisada em intervalos de $0,5 \mathrm{~mm} /$ dia. Após análise dos dados obtidos, verificou-se que a maior frequência de ocorrência foi entre 5,0 e $5,5 \mathrm{~mm} /$ dia com $25 \%$. O intervalo entre 3,5 e $5,5 \mathrm{~mm} /$ dia apresentou uma frequência de ocorrência de $76,7 \%$.

Foi possível observar que no intervalo entre 3,5 e $5,5 \mathrm{~mm} /$ dia concentra-se uma amostra considerável das evapotranspirações de referência em Januária (MG), como pode se ver na Fig. 10.

Através dos modelos apresentados, pode-se concluir que a evapotranspiração para a cidade de Januária, pode ser determinada conhecendo-se a radiação líquida ou a temperatura do ar com um bom grau de confiabilidade. 
Santos Madureira, R.; Antunes de Macêdo, J; Martins da Silva Castro, A.

\section{Referências}

[1] R. G. Allen; L. S. Pereira; D. Raes and M. Smith. Crop evapotranspiration: guidelines for computing crop water requirements. FAO. Irrigation and Drainage Paper, 56. Rome: FAO, 1998, 297p.

[2] J. C. Barbosa. Modelagem na Educação Matemática: Uma perspectiva. In: I Encontro Paranaense De Modelagem Em Educação Matemática, 2004, Londrina. Anais. Londrina: UEL, 2004. 1 CD-ROM.

[3] R. C. Bassanezi. Ensino-aprendizagem com modelagem matemática: uma nova estratégia. São Paulo: Contexto, 2002.

[4] M. A. Berlato e L. C. B. Molina. Evaporação e evapotranspiração. Porto Alegre: IPAGRO. Boletim Técnico, n.7, 1981. 95 p.

[5] E. Bisognin; V. Bisognin e O. A. Rays. Modelo matemático da concentração de cocaína no organismo humano: Modelagem Matemática no ensino de Matemática. Educação Matemática em Revista - RS, n. 6, 2004, Ano VI. SBEM, RS.

[6] M. Cargnin-Stieler e V. Bisognin. Radiação solar ultravioleta e a Modelagem Matemática. In: IX Encontro Gaúcho de Educação Matemática, 2006, Caxias do Sul. Anais. Caxias do Sul: 2006.

[7] CPTEC - Centro de Previsão de Tempo e Estudos Climáticos (CPTEC) do Instituto Nacional de Pesquisas Espaciais (INPE). Cachoeira Paulista (SP), 2014. Disponível em: http: www.cptec.inpe.br/. Acesso em 02 abr. 2020.

[8] J. Doorenbos and J. O. Pruitt. Crop water requirement. Rome: FAO, 1977. 144 p.

[9] K. Reichardt. A água em sistemas agrícolas. São Paulo: Manole LTDA, 1990. 188 p.

Submetido em 22 de Abril de 2020. Aceito em 10 de Agosto de 2020. 